\title{
Integrands and loop momentum in string and field theory
}

\author{
Piotr Tourkine $\odot^{*}$ \\ CNRS, LPTHE, Sorbonne Université, 4 place Jussieu, 75005 Paris, France
}

(Received 4 June 2020; accepted 15 June 2020; published 8 July 2020)

\begin{abstract}
The notion of a unique integrand does not a priori makes sense in field theory: different Feynman diagrams have different loop momenta and there should be no reason to compare them. In string theory, however, a global integrand is natural and allows one, for instance, to make explicit the separation between left- and right-moving degrees of freedom. However, the significance of this integrand had not really been investigated so far. It is even more important in view of the recently discovered loop monodromies that are related to the duality between color and kinematics in gauge and gravity loop amplitudes. This paper intends to start filling this gap, by presenting a careful definition of the loop momentum in string theory and describing precisely the resulting global integrand obtained in the field-theory limit. We will then apply this technology to write down some monodromy relations at two and three loops and make contact with the color-kinematics duality.
\end{abstract}

DOI: 10.1103/PhysRevD.102.026006

\section{INTRODUCTION}

In the last few years, a variety of results for scattering amplitudes in field theory at loop level have been derived using string theoretic methods. Interestingly, many of them have focused on integrands and have involved explicit dependence on a loop momentum defined globally for a string integrand.

While this is a peculiar idea from a traditional Feynman perspective, this concept is actually present, though maybe not emphasized, since the very early days of string theory [1]. The seminal papers [2-5] then laid the foundations for the definition of the loop momentum in string theory amplitudes in their modern formulation as conformal field-theory correlation functions integrated over the moduli space of Riemann surfaces. Those correlation functions can be written as holomorphic squares in loop amplitudes only in the presence of loop momentum. Especially nontrivial for superstrings (in the Ramond-Neveu-Schwarz formulation), this property was called chiral splitting.

However, some aspects related to the precise definition of the loop momentum had not been worked out and the recent results alluded to above require us to now reinvestigate this question. We have especially in mind two categories of results: the monodromy relations at higher loops in string theory derived in $[6,7]$ and the scattering

*piotr.tourkine@cern.ch

Published by the American Physical Society under the terms of the Creative Commons Attribution 4.0 International license. Further distribution of this work must maintain attribution to the author(s) and the published article's title, journal citation, and DOI. Funded by SCOAP ${ }^{3}$. equation or ambitwistor string methods at loop level. This paper will be focused on the former; we allude to the latter in the discussion.

The monodromy relations in string theory were originally derived at tree level [8-10]. They are now understood to generalize of the Bern-Carrasco-Johansson (BCJ) [11] duality between color and kinematics that underlie the so-called double-copy construction [12] of gravity integrands as squares of Yang-Mills integrands. While implemented very efficiently to compute loop amplitudes (see for instance the last recent achievement at five loops [13] and the review [14]), this duality is still not understood from first principles.

The tree-level relations were extended to all loop orders in $[6,7]$ in open-string theory, which generalized some previous works in field theory [15-19]. This gives hope that string theory can shed light on the colorkinematics duality and these relations need to be understood deeper. In particular, some aspects related to the definition of the loop momentum were only conjectured in [6] and the present paper intends to fill this gap and show in detail how to apply the monodromy relations at higher loops.

Another aspect that this paper deals with is the notion of an integrand in field theory. In [6], it was emphasized that the relations induced in field theory by the stringy monodromies are valid globally at the integrand level, i.e. mix different integrands of different graphs at the same value of the loop momentum, as in [20]. We will see how this picture generically emerges from the field-theory limit of string amplitudes.

Here is a summary of the main contributions of this paper. 


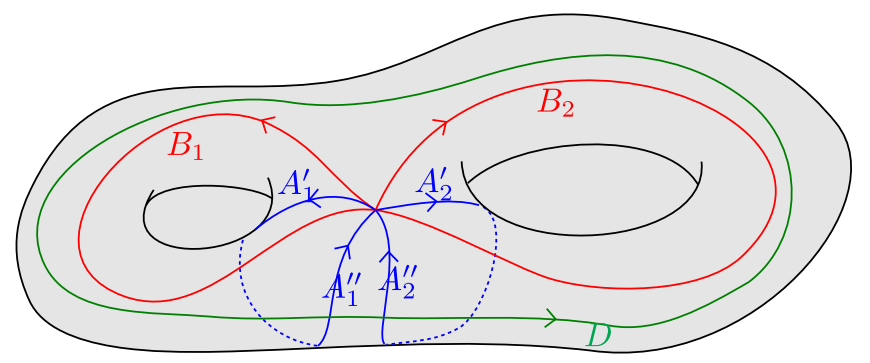

FIG. 1. Canonical dissection along the homology cycles.

Firstly, we give a precise definition of the loop momentum in the string theory integrand in Sec. II, from a review of classic computations and from solving directly the classical equations of motion for the string. The definition requires working on a so-called canonical dissection of the surface (see Fig. 1), which, importantly, breaks modular invariance [4] because it does not allow one to modify the homology basis anymore after the loop momentum is integrated out and the invariance is restored.

Secondly, we provide a careful study of its field-theory limit (in Sec. II), which as a by-product gives how the loop momentum is distributed across all Feynman graphs appearing in this limit. This analysis uses some tools to study the degeneration of Riemann surfaces.

Finally, we provide applications of these definitions in loop amplitudes. In particular, we shall see in detail how the monodromy relations work two- and three-loop amplitudes, which support further the claim that the monodromy relations generalize the BCJ duality. More precisely it will support the conjecture that in all higher loop relations, the monodromy relations always combine the numerators appearing in the field-theory limit into groups of graphs called BCJ triplets.

It should be noted that in this paper we will exclusively be concerned with the bosonic part of the string amplitudes, which is the one that carries the loop-momentum zero modes. Further applications of these results are presented in the discussion in Sec. V together with open questions.

\section{STRING THEORY}

The presence of loop momentum is standard in the operator formalism of the string theory; this is for instance how amplitudes are derived in the classic textbook by Green, Schwarz and Witten [21]. These representations have the advantage to make chiral splitting manifest; i.e. the string integrand is factorized as a product of a purely left-moving (holomorphic) and right-moving (antiholomorphic) part. The traditional form of the string amplitudes is obtained after integrating out the loop momentum, which induces nonholomorphy in the integrand and destroys its chiral splitting.

The drawback, however, is that this formalism is difficult to use at high multiplicity and loop orders because it amounts to doing a very complicated Feynman diagram computation, and the number of graphs increases quickly. Besides, the structure of the moduli space of Riemann surfaces at higher genus essentially renders the whole process unusable. The modern approach to string theory scattering amplitudes is based on complex (super)geometry and conformal field-theory techniques [4]. In this manner, the nonholomorphic terms are generated from the start [2-5], essentially because meromorphic functions on Riemann surfaces must have the sum of their residues vanishing, via Stoke's theorem [22].

We will now review the construction of the universal part to string theory amplitudes at loop level. It is the generalization of the Koba-Nielsen factor $\prod_{i<j} \mid z_{i}-$ $\left.z_{j}\right|^{\alpha^{\prime} k_{i} \cdot k_{j}}$ ubiquitous to tree-level string amplitudes. Here and throughout, $z_{i}$ will be the locations of the vertex operators on the string world sheet, $\alpha^{\prime}$ is the string Regge slope, and $k_{i}$ are null momenta of the states, all taken to be incoming, that satisfy momentum conservation $\sum_{i=1}^{n} k_{i}=0$ for and $n$-particle process.

Along the way we shall see how the loop momentum appears. We will mostly follow [4] and supplement the construction with careful normalizations and definitions of the loop momentum. Note that the paper [23] presents details on these computations and an exhaustive reference list on the matter.

In the conformal gauge, the Polyakov action for closed strings reads

$$
S_{\mathrm{P}}=\frac{1}{2 \pi \alpha^{\prime}} \int_{\Sigma} \partial_{z} X \partial_{\bar{z}} X
$$

where $X^{\mu}(z, \bar{z})$ are the coordinates of the string in $d$-dimensional target flat space.

The equations of motion of the theory without vertex operator insertions split the $X$ field into left and right movers as

$$
X^{\mu}(z, \bar{z})=X_{L}^{\mu}(z)+X_{R}^{\mu}(\bar{z}),
$$

which will share a common zero mode $x_{L}=x_{R}$ and a loopmomentum zero mode to be introduced momentarily. In the presence of $n$ plane-wave vertex operator insertions [24]

$$
V_{j}\left(k_{j}\right)=\exp \left(i k_{j} \cdot X\left(z_{j}, \bar{z}_{j}\right)\right)
$$

the phases can be inserted in the action and the object we seek to compute is given by

$$
\begin{aligned}
& \left\langle\left\langle V_{1}\left(k_{1}\right) \ldots V_{n}\left(k_{n}\right)\right\rangle\right\rangle \\
& =\int \mathcal{D} X e^{-\left(1 / 2 \pi \alpha^{\prime}\right) \int d^{2} z \partial_{z} X^{\mu} \partial_{\bar{z}} X_{\mu}+2 i \pi \alpha^{\prime} \sum_{j=1}^{n} k_{j}^{\mu} X_{\mu}(z, \bar{z}) \delta^{2}\left(z-z_{i}\right) d^{2} z},
\end{aligned}
$$


where the double bracket notation is that of [4]. To compute this path integral, we need to invert the kinetic operator $\partial_{z} \partial_{\bar{z}}$, i.e. compute the Green's function

$$
G(z, w) \eta^{\mu \nu}=\left\langle X(z, \bar{z})^{\mu} X(w, \bar{w})^{\nu}\right\rangle
$$

The subtlety when doing this directly comes from the fact that $\partial_{z}$ and $\partial_{\bar{z}}$ have zero modes on a compact Riemann surface $\Sigma$ of genus $g \geq 1$, that correspond to loop momentum. They are supported by $g$ holomorphic and antiholomorphic one-forms $\omega_{I}$ and $\bar{\omega}_{J}$ that span the cohomologies $H^{(1,0)}(\Sigma)$ and $H^{(0,1)}(\Sigma)$ :

$$
\forall I=1 \ldots g, \quad \partial \bar{\omega}_{I}=0, \quad \bar{\partial} \omega_{I}=0 .
$$

In this equation and below, $\partial$ and $\bar{\partial}$ are operators $\partial=(\partial / \partial z) d z$ and $\bar{\partial}=(\partial / \partial \bar{z}) d \bar{z}$, as defined in the Appendix. We also abbreviate $\partial_{z}:=(\partial / \partial z)$ and likewise for $\partial_{\bar{z}}$.

The holomorphic one-forms are dual to a homology of one-cycles, traditionally called $a$ - and $b$-cycles, canonically defined by their intersection numbers $a_{I} \cap b_{J}=\delta_{I, J}$, for $I, J=1 \ldots g$, all other vanishing. Pairing a cycle with a form is done via the period map $(\omega, c) \mapsto \int_{c} \omega$. Normalizing the period of the one-forms on the $a_{I}$-cycles to $\delta_{I J}$ makes the periods along the $b$-cycles define the period matrix $\Omega$ of the surface as follows:

$$
\oint_{a_{I}} \omega_{J}=\delta_{I J}, \quad \oint_{b_{I}} \omega_{J}=\Omega_{I J} .
$$

It is a symmetric $g \times g$ matrix with positive-definite imaginary part $\operatorname{Im} \Omega>0$.

Let us then consider a Riemann surface $\Sigma$ of genus $g$. The kinetic operator can be inverted on the space orthogonal to the zero modes [2-4] and the equations that define the corresponding Green's function are

$$
\begin{gathered}
\int_{\Sigma} G(z, w) d^{2} z=0, \\
\partial_{z} \partial_{\bar{z}} G(z, w)=-2 \pi \alpha^{\prime} \delta^{2}(z-w)+\frac{2 \pi \alpha^{\prime}}{\int d^{2} z \sqrt{g}}, \\
\partial_{z} \partial_{\bar{w}} G(z, w)=2 \pi \alpha^{\prime} \delta^{(2)}(z-w) \\
-\alpha^{\prime} \pi \sum_{I, J} \omega_{I}(z)(\operatorname{Im} \Omega)_{I J}^{-1} \bar{\omega}_{J}(w),
\end{gathered}
$$

where $g$ is the determinant of the metric on the surface, as defined in the Appendix. These equations can be solved and yield

$$
\begin{aligned}
G\left(z_{1}, z_{2}\right)= & -\frac{\alpha^{\prime}}{2} \ln \left(\left|E\left(z_{1}, z_{2}\right)\right|^{2}\right) \\
& +\alpha^{\prime} \pi \operatorname{Im}\left(\int_{z_{2}}^{z_{1}} \omega_{I}\right)\left((\operatorname{Im} \Omega)^{-1}\right)^{I J} \operatorname{Im}\left(\int_{z_{2}}^{z_{1}} \omega_{J}\right)
\end{aligned}
$$

up to terms which we neglect because vanish on the support of momentum conservation. The prime form $E$ is defined in Eq. (A8). Its essential property is that it vanishes linearly on the diagonal

$$
E(x, y)=x-y+O(x-y)^{3} .
$$

It is defined on the universal cover of $\Sigma$, because it has monodromies [given in Eq. (A10)] along $a$ - and $b$-cycle transportation. The nonholomorphic correction in Eq. (11) exactly cancels these monodromies and the Green's function is correctly defined on the surface and not its cover.

The correlation function (4) is then computed by Wick's theorem:

$$
\left\langle\prod_{i=1}^{n} e^{i k_{i} X\left(z_{i}, \bar{z}_{i}\right)}\right\rangle=e^{-\sum_{i<j} k_{i} \cdot k_{j} G\left(z_{i}, z_{j}\right)} .
$$

Because of the nonholomorphic terms, this expression cannot be written as it stands as a modulus square. Note that they are absent at tree level:

$$
\left\langle X^{\mu}\left(z_{1}, \bar{z}_{1}\right) X^{\nu}\left(z_{2}, \bar{z}_{2}\right)\right\rangle=-\frac{\alpha^{\prime}}{2} \eta^{\mu \nu} \ln \left(\left|z_{1}-z_{2}\right|^{2}\right)
$$

and the correlator (12) can be chirally split. At loops, where the $\ln |E|^{2}$ term similarly poses no problem, in the exponential of (12) the problematic terms are

$Q_{N H}=\alpha^{\prime} \pi \sum_{i<j} k_{i} \cdot k_{j} \operatorname{Im}\left(\int_{z_{j}}^{z_{i}} \omega_{I}\right)\left((\operatorname{Im} \Omega)^{-1}\right)^{I J} \operatorname{Im}\left(\int_{z_{j}}^{z_{i}} \omega_{J}\right)$.

Let $P$ be a point on the surface, so that we can decompose the integration $\int_{z_{j}}^{z_{i}}$ as $\int_{z_{j}}^{P}+\int_{P}^{z_{i}}$, and (14) then becomes

$$
\begin{aligned}
Q_{N H}= & \alpha^{\prime} \pi \sum_{i<j} k_{i} \cdot k_{j} \operatorname{Im}\left(\int_{z_{j}}^{P}+\int_{P}^{z_{i}} \omega_{I}\right)\left((\operatorname{Im} \Omega)^{-1}\right)^{I J} \\
& \times \operatorname{Im}\left(\int_{z_{j}}^{P}+\int_{P}^{z_{i}} \omega_{J}\right) .
\end{aligned}
$$

The diagonal terms $\operatorname{Im} \int_{P}^{z_{i}} \omega_{I}\left((\operatorname{Im} \Omega)^{-1}\right)^{I J} \operatorname{Im} \int_{P}^{z_{i}} \omega_{J}$ vanish by momentum conservation (summing over $j$ in this case), so we keep only the crossed terms and we would want to rewrite (15) as 


$$
\begin{aligned}
Q_{N H}= & -2 \pi \alpha^{\prime} \sum_{i<j} k_{i} \cdot k_{j} \operatorname{Im}\left(\int_{P}^{z_{j}} \omega_{I}\right)\left((\operatorname{Im} \Omega)^{-1}\right)^{I J} \\
& \times \operatorname{Im}\left(\int_{P}^{z_{i}} \omega_{J}\right)
\end{aligned}
$$

(the sign comes from flipping the orientation of the integration in one term). The reason why this identity is not straightforward is because it is valid if and only if all the paths from $P$ to $z_{i}$ need to be uniquely defined. Hence, we are looking for a way to define uniquely, for all values of $z_{i}$ on $\Sigma$, a path from $P$ to $z_{i}$. Ambiguities can arise from $z$ winding along a nontrivial cycle, and therefore what we describe is a way to cut open the Riemann surface into a polygon with $4 g$ faces, called its canonical dissection, as in Fig. 1. It is defined by cutting open the surface along the $a$ - and $b$-cycles, not considered anymore as representatives in the homology, but as actual curves, all of which touch in one point exactly.

Now, because the sum is factorized in (16), we can introduce a Gaussian $d$-dimensional integration so that

$$
\begin{gathered}
(\operatorname{det} \operatorname{Im} \Omega)^{d / 2} \int \frac{d^{D} \ell}{(2 \pi)^{D}} e^{-\pi \alpha^{\prime} \ell_{I} \ell_{J} \operatorname{Im} \Omega^{I J}-2 \pi \alpha^{\prime} \sum_{i, J} \ell_{J} \cdot k_{i} \operatorname{Im} \int_{P}^{z_{i}} \omega_{J}} \\
=e^{2 \pi \alpha^{\prime} \sum_{i<j} k_{i} \cdot k_{j} \operatorname{Im}\left(\int_{P}^{z_{j}} \omega_{I}\right)\left((\operatorname{Im} \Omega)^{-1}\right)^{I J} \operatorname{Im}\left(\int_{P}^{z_{i}} \omega_{J}\right)}
\end{gathered}
$$

up to a global normalization factor. Using that $\exp (-2 \operatorname{Im} x)=|\exp (x)|^{2}$, this expression can be further rewritten as a modulus square, and finally we have

$$
\left\langle\left\langle V_{1}\left(k_{1}\right) \ldots V_{n}\left(k_{n}\right)\right\rangle\right\rangle=\int \frac{d^{d} \ell}{(2 \pi)^{d}}\left\langle\left\langle V_{1}\left(k_{1}\right) \ldots V_{n}\left(k_{n}\right)\right\rangle\right\rangle\left(\ell_{I}\right),
$$

where

$$
\begin{aligned}
& \left\langle\left\langle V_{1}\left(k_{1}\right) \ldots V_{n}\left(k_{n}\right)\left(\ell_{I}\right)\right\rangle\right\rangle \\
& =(\operatorname{Im} \Omega)^{d / 2} \mid e^{\left(i \pi \alpha^{\prime} / 2\right) \ell_{I} \ell_{J} \Omega^{I J}+i \pi \alpha^{\prime} \sum_{i, J} \ell_{J} \cdot k_{i} \int_{P}^{z_{i}} \omega_{J}} \\
& \quad \times\left.\prod_{i, j} E\left(z_{i}, z_{j}\right)^{\alpha^{\prime} k_{i} k_{j} / 2}\right|^{2}
\end{aligned}
$$

which is Eq. (2.99) of [4]. This is the content of chiral splitting for the bosonic part of the amplitudes.

The most important conclusion of this section is that the loop momenta are defined with respect to a specific canonical dissection, and not just the homology. Now we will see how this can be derived from looking at the classical trajectory for the field $X$; this will lead to a precise definition of the momentum flowing through a given cycle.

A consequence of working on a canonical dissection is that modular invariance (the freedom to change $a$ - and $b$-cycles) is totally broken, because $a$ - and $b$-cycles cannot be mixed anymore within the string integrand. Of course, reintegrating out the loop momentum gives modular invariant expressions.

Classical solution.-Since the action is free, all those quantities could have been equivalently computed from the classical solution of the Euler-Lagrange equation with sources. For $X$, it can be obtained by varying the action (4) or, equivalently, by computing

$$
X_{\text {class }}^{\mu}(z, \bar{z})=\frac{\left\langle X^{\mu}(z, \bar{z}) \prod_{i=1}^{n} e^{i k_{i} X\left(z_{i}, \bar{z}_{i}\right)}\right\rangle}{\left\langle\prod_{i=1}^{n} e^{i k_{i} X\left(z_{i}, \bar{z}_{i}\right)}\right\rangle}
$$

using the individual two-point functions $\langle X X\rangle$.

Let us follow the former approach. We want to minimize the following action:

$S=\frac{1}{2 \pi \alpha^{\prime}} \int_{\Sigma} \partial_{z} X \partial_{\bar{z}} X+2 i \pi \alpha^{\prime} \sum_{j} k_{j}^{\mu} X(z, \bar{z}) \delta^{2}\left(z-z_{i}, \bar{z}-\bar{z}_{i}\right)$.

It is instructive to first do the computation at tree level when there are not yet zero modes. The $\frac{\delta}{\delta X}$ variation of this Lagrangian yields

$$
2 \partial_{z} \partial_{\bar{z}} X^{\mu}=2 i \pi \alpha^{\prime} \sum_{i} k_{i}^{\mu} \delta^{2}\left(z-z_{i}, \bar{z}-\bar{z}_{i}\right) .
$$

Using that

$$
\partial_{\bar{z}} \frac{1}{z}=\partial_{z} \frac{1}{\bar{z}}=2 \pi \delta^{(2)}(z, \bar{z}),
$$

this integrates once to

$$
\partial_{\bar{z}} X^{\mu}=\frac{i \alpha^{\prime}}{2} \sum_{i} \frac{k_{i}^{\mu}}{\bar{z}-\bar{z}_{i}}
$$

and then

$$
X_{\text {class }}^{\mu}=x_{R}+\frac{i \alpha^{\prime}}{2} \sum_{i} k_{i}^{\mu} \ln \left(\bar{z}-\bar{z}_{i}\right)+X_{L}(z) .
$$

The holomorphic part is determined by reinjecting this equation in the equations of motion and one finds

$$
X_{\text {class }}^{\mu}=x_{0}+\frac{i \alpha^{\prime}}{2} \sum_{i} \ln \left|z-z_{i}\right|^{2},
$$

where $x_{0}=x_{L}+x_{R}$ is the zero mode that gives rise to momentum conservation upon $\int d^{d} x_{0}$ integration.

Let us now go to loop level and consider a Riemann surface $\Sigma$ of genus $g$. Analogously to the tree-level case, we can obtain the singular part of $\partial X_{L}^{\mu}(z)$ in terms of meromorphic differentials with single poles $\omega_{z_{+}, z_{-}}=$ $\omega_{z_{+}, z_{-}}(z) d z$ with residue \pm 1 at $z=z_{ \pm}$. They are called 
Abelian differentials of third kind [25] and can be uniquely defined by normalizing to zero their periods along the $a$-cycles:

$$
\omega_{z_{+}, z_{-}}(z) \underset{z \rightarrow z_{ \pm}}{\sim} \pm \frac{1}{z-z_{ \pm}} ; \quad \forall I=1 \ldots g, \quad \oint_{a_{I}} \omega_{z_{+}, z_{-}}=0 .
$$

For further convenience, let us denote $c_{i}$ the circles $\left|z-z_{i}\right|=\epsilon$. This allows us to define a singular homology on $\Sigma-\left\{z_{1}, \ldots, z_{n}\right\}$ by augmenting that of $\Sigma$ with these $n$ new $c$-cycles.

The new ingredient compared to the tree-level case is the presence of zero modes for the $\bar{\partial}$ and $\partial$ operators, given by the holomorphic one-forms and their complex conjugates, as in (6). After the first integration of (22), we find

$$
\begin{aligned}
& \partial X_{L}^{\mu}(z)=i \pi \alpha^{\prime} \sum_{J=1}^{g} \omega_{J} \ell_{J}^{\mu}+\frac{i \alpha^{\prime}}{2} \sum_{i=1}^{n} \omega_{z_{i}, z_{0}} k_{i}^{\mu}, \\
& \bar{\partial} X_{R}^{\mu}(\bar{z})=i \pi \alpha^{\prime} \sum_{J=1}^{g} \bar{\omega}_{J} \tilde{\ell}_{J}^{\mu}+\frac{i \alpha^{\prime}}{2} \sum_{i=1}^{n} \bar{\omega}_{z_{i}, z_{0}} k_{i}^{\mu},
\end{aligned}
$$

where $z_{0}$ is an extra variable whose dependence drops out by momentum conservation. We left unspecified the zero modes for the holomorphic and antiholomorphic fields; they will be fixed later by a physical requirement to measure a correctly normalized momentum. Integrating once more gives

$$
X_{L, \text { class }}^{\mu}(z)=x_{L}^{\mu}+i \pi \alpha^{\prime} \sum_{J=1}^{g} \ell_{J}^{\mu} \int_{P}^{z} \omega_{J}+\frac{i \alpha^{\prime}}{2} \sum_{i=1}^{n} k_{i}^{\mu} \int_{P}^{z} \omega_{z_{i}, z_{0}},
$$

$$
X_{R, \text { class }}^{\mu}(\bar{z})=x_{R}^{\mu}+i \pi \alpha^{\prime} \sum_{J=1}^{g} \tilde{e}_{J}^{\mu} \int_{P}^{\bar{z}} \bar{\omega}_{J}+\frac{i \alpha^{\prime}}{2} \sum_{i=1}^{n} k_{i}^{\mu} \int_{P}^{\bar{z}} \bar{\omega}_{z_{i}, z_{0}} .
$$

Finally, $X_{\text {class }}^{\mu}$ is given by the sum of these two equations. To make contact with the previous derivation and Eq. (19) in particular, note that the prime form is related to the Abelian differentials of the third kind by

$$
\partial_{z} \ln \left(\frac{E(z, a)}{E(z, b)}\right)=\omega_{a, b}(z) .
$$

This also defines uniquely the zero modes of $\partial X, \bar{\partial} X$ with correct normalization. To measure the loop momentum flowing through a typical cycle $C$, which is a combination of the canonical $a_{J}$-cycles and $c_{i}$-cycles, we define the following flux:

$$
P_{C}^{\mu}=\frac{1}{2 \pi \alpha^{\prime}} \oint_{C}\left(-\partial_{z} d z+\partial_{\bar{z}} d \bar{z}\right) X
$$

The normalization is fixed in a first stage by demanding that integration along $c_{i}$-cycles provides momentum $k_{i}$ :

$$
\begin{aligned}
\frac{1}{2 \pi \alpha^{\prime}} \oint_{c_{i}}\left(-\partial_{z} d z+\partial_{\bar{z}} d \bar{z}\right) X^{\mu} & =k_{i}^{\mu} \frac{i \alpha^{\prime}}{4 \pi \alpha^{\prime}} \oint_{c_{i}}\left(-\omega_{z_{i}, z_{0}}+\bar{\omega}_{z_{i}, z_{0}}\right) \\
& =k_{i}^{\mu} .
\end{aligned}
$$

Then we have

$$
\frac{1}{2 \pi \alpha^{\prime}} \oint_{a_{I}}\left(-d z \partial_{z}+d \bar{z} \partial_{\bar{z}}\right) X^{\mu}=-\delta_{I J} i\left(\ell_{J}^{\mu}-\tilde{\ell}_{J}^{\mu}\right) / 2 \equiv \ell_{I}^{\mu}
$$

if the loop momenta are taken to be purely imaginary. This derivation gives another check of this property which was originally observed in $[4,5]$ and that seems fundamental to string theory on euclidean world sheets. It would be interesting to study the consequences of this fact in the ambitwistor string where a similar normalization was observed to arise by matching against field-theory computations in [26].

Open strings on orientable surfaces are obtained by modding out by the involution $z \simeq \bar{z}$ along the $a$-cycles of the string world sheet [27] and letting the punctures live on the boundary of the surfaces. More precisely, if $z=\rho e^{i \theta}$ with $\theta \in[0,2 \pi[$ is a local coordinate along an $a$-cycle, we identify $\theta \leftrightarrow-\theta$. This is the natural involution to describe the gauge theory channel of open-string amplitudes, which we will use later to apply the monodromy relations in openstring theory and their induced relations in field theory. This involution can also be used to obtain some nonplanar graphs, as long as they are given by orientable surfaces.

Note also that this turns the cycles of the canonical dissection into segments on the world sheet such that $\oint_{a_{I}} \omega_{J} \rightarrow \int_{a_{I}^{\prime}} \omega_{J}=\delta_{I J} / 2$, where $a_{I}^{\prime}$ is $a_{I}$ modulo the involution.

\section{FIELD-THEORY INTEGRAND}

In this section we will investigate one implication of the previous considerations. Since there exists a global integrand in string theory, there needs to exist one in field theory, induced via the field-theory limit. In practice, after studying the field-theory limit itself, we will be able to describe the graph integrand topologies: external leg ordering and labeling of the internal loop momenta.

The understanding of the mechanism of the field-theory limit of string graphs is almost as old as string theory itself [28]. It is produced by corners of the moduli space where the surface degenerates so that all internal edges become infinitely long and thin (this is a $b$-cycle statement) or 


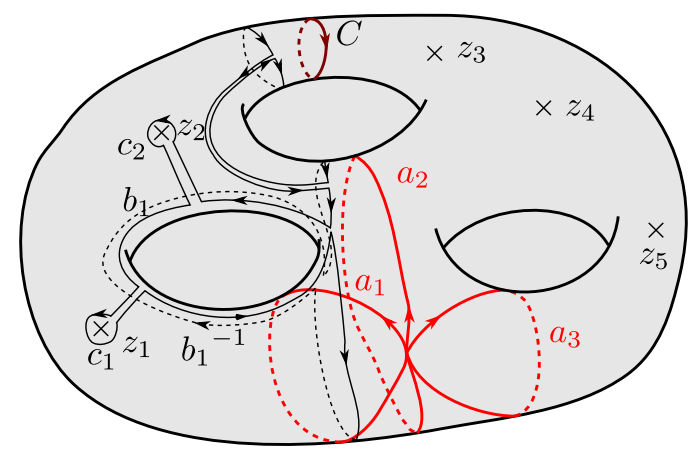

FIG. 2. From the picture we see that $C \cup\left(a_{2}\right)^{-1} \cup b_{1} \cup$ $\left(b_{1}\right)^{-1} \cup c_{1} \cup c_{2}=\mathrm{id}$; hence, $C=a_{2} \cup\left(c_{1}\right)^{-1} \cup\left(c_{2}\right)^{-1}$ and the momentum flowing through $C$ is given by $\frac{1}{2 \pi \alpha^{\prime}} \oint_{C}(-\partial+\bar{\partial}) X^{\mu}=$ $\ell_{2}^{\mu}-k_{1}^{\mu}-k_{2}^{\mu}$.

equivalently where all $a$ - and $c$-cycles are pinched. This is a continuous process, known in the math literature as a tropical limit [29].

The property which we will need to describe the graph loop-momentum labeling is that the momentum flowing through a cycle is preserved by the field-theory limit. As the momentum is a zero mode, it is not affected by the decoupling of the excited states of the string; therefore, the result which we seek is physically sound and the problem reduces to a computational matter. Let $C$ be a closed curve made of $a_{I^{-}}$and $c_{i}$-cycles:

$$
C=\cup_{i \in \mathcal{I}_{C}} \mathbf{a}_{i}
$$

where $\mathbf{a}$ is either an $a$-cycle or a $c$-cycle with coefficient 1 . This defines implicitly the set $\mathcal{I}_{C}$. This excludes the possibility that our cycle $C$ could wind multiple times. For illustrative purposes, see Fig. 2. Let us call the corresponding momentum

$p_{C}^{\mu}=-\frac{1}{2 \pi \alpha^{\prime}} \oint_{C}\left(d z \partial_{z}-d \bar{z} \partial_{\bar{z}}\right) X=\sum_{I, i \in \mathcal{I}_{C}}\left(\ell_{I}^{\mu}+k_{i}^{\mu}\right)$

with obvious notations for the summation. The crucial point is that this quantity is a topological invariant; therefore, it cannot change as we deform continuously the surface when taking the field-theory limit. We now will check this property, see when the $C$ cycle degenerates, and demonstrate that a propagator $1 / p_{C}^{2}$ factorizes out of the string amplitude.

\section{A. Single pinching of a Riemann surface}

There are two types of degenerations that a Riemann surface can undergo: separating and nonseparating. The separating degeneration corresponds to pinching off a trivial cycle in the homology or a $c$-cycle: it splits apart a surface of genus $g$ into two surfaces of genera $g_{1}$ and $g_{2}$ such that $g=g_{1}+g_{2}$. A nonseparating degeneration

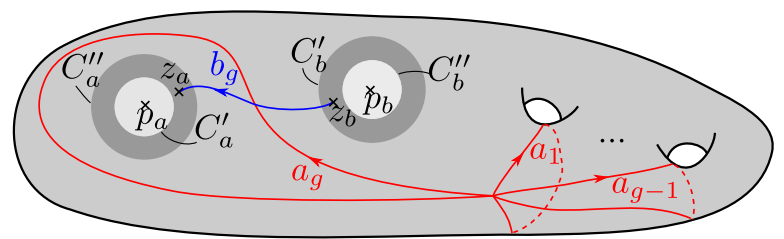

FIG. 3. Handle-representation drawing of the pinching that we studied in this section.

pinches off an $a$-type cycle and the resulting object is a surface with genus decreased by one unit and two extra punctures. This is the case of interest for us because we want to check that a propagator with expected loopmomentum labeling is generated. An example of such a degeneration is provided in Fig. 3.

Firstly, let us observe that we do not lose in generality by considering that the $a$-cycle part of our $C$ cycle is the cycle $a_{g}$ (we could always relabel the $a$-cycles).

The degeneration of the Riemann surface is done via the so-called plumbing fixture construction; see [30] or [4,31]. In this construction, the degenerating curve $\Sigma_{g}$ is constructed from a Riemann surface of genus $g-1, \Sigma_{g-1}$ with period matrix $\Omega_{g-1}$ and a pair of points marked on the surface $p_{a}$ and $p_{b}$; see Fig. 4. To construct $\Sigma_{g}$, one constructs two pairs of circles centered around $p_{a}$ and $p_{b}: C_{a}^{\prime \prime}$ and $C_{b}^{\prime}$ of radius 1 and $C_{a}^{\prime}, C_{b}^{\prime \prime}$ of radius $|q|<1$ for complex number $q$ that parametrizes the degeneration. The internal disk is then cut out of the surface and the annuli between the disks are identified via an invertible map

$$
x y=q .
$$

The extra $a$-cycle $a_{g}$ is a closed loop around $C_{a}^{\prime \prime}$ for instance; the extra $b$-cycle $b_{g}$ is a line that connects any two points $z_{a}$ and $z_{b}$ in the annuli that obey $z_{a} z_{b}=q$. Choosing $\left|z_{a}\right|=\left|z_{b}\right|=\sqrt{|q|}$ ensures that when $q=0$, the extra cycle is really the line connecting the two points $p_{a}$ and $p_{b}$ which are identified. Then, if $\Omega_{g}$ is the period matrix of $\Sigma_{g}$, Fay in [30] proves that

$$
\Omega_{g} \sim\left(\begin{array}{c|c}
\Omega_{g-1} & \vec{v} \\
\hline \vec{v}^{t} & \tau
\end{array}\right), \quad \text { where } q=e^{2 i \pi \tau},
$$

up to subleading terms and where the components of $\vec{v}$ are given by $v_{I}=\int_{p_{a}}^{p_{b}} \omega_{I}^{(g-1)}, I=1 \ldots g-1$. The exponent on

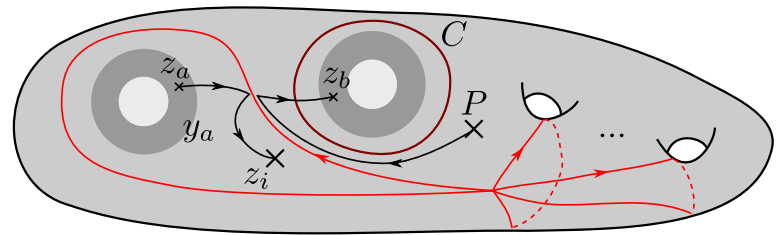

FIG. 4. Illustration of the plumbing fixture construction. 
the differential form $\omega_{I}^{(h)}$ designates the surface $\Sigma_{h}$ to which it is associated for $h=g, g-1$.

With this, we can already extract the degeneration of the quadratic term in the loop momentum in the exponential in Eq. (19):

$$
\begin{aligned}
\sum_{I, J=1}^{g} \ell_{I} \cdot \ell_{J} \operatorname{Im} \Omega_{g}= & \ell_{g}^{2} \operatorname{Im} \tau+2 \sum_{I=1}^{g-1} \ell_{I} \cdot \ell_{g} \operatorname{Im}\left(v_{I}\right) \\
& +\sum_{I, J=1}^{g-1} \ell_{I} \cdot \ell_{J} \operatorname{Im} \Omega_{g-1} .
\end{aligned}
$$

To study the degeneration of the other two terms in (19), we need the degeneration of the differential forms oneforms. That of the holomorphic forms is standard and detailed in the references mentioned above:

$$
\begin{aligned}
& \omega_{I}^{(g)}=\omega_{I}^{(g-1)}+O(q), \\
& \omega_{g}^{(g)}=\omega_{p_{a}, p_{b}}^{(g-1)}+O(q) .
\end{aligned}
$$

This allows us to extract the degeneration of the second term in (19):

$$
\begin{aligned}
\sum_{i, J} \ell_{J} \cdot k_{i} \int_{P}^{z_{i}} \omega_{J}^{(g)}= & \ell_{g} \cdot \sum_{i=1}^{n} k_{i} \int_{P}^{z_{i}} \omega_{p_{a}, p_{b}}^{(g-1)} \\
& +\sum_{i=1}^{n} \sum_{J=1}^{g-1} \ell_{J} \cdot k_{i} \int_{P}^{z_{i}} \omega_{J}^{(g-1)}+O(q) .
\end{aligned}
$$

We therefore need to evaluate the integrals $\int_{P}^{z_{i}} \omega_{p_{a}, p_{b}}^{(g-1)}$. The circle cycle $C$ defined as above is represented in Fig. 5. It cuts out the surface into two distinct components (we are still working in the canonical dissection; hence, one should not cross through the $a$-cycles).

When $P$ and $z_{i}$ are on the same side, almost nothing is to be done and $\int_{P}^{z_{i}} \omega_{p_{a}, p_{b}}^{(g-1)}$ provides directly two terms similar to the $k_{i} \cdot k_{j}$ terms of Eq. (19). To see this, we use the reciprocity theorem [32] for Abelian differentials of the third kind with zero $a$ periods:

$$
\int_{B}^{A} \omega_{C, D}=\int_{D}^{C} \omega_{A, B} .
$$

Therefore we have that
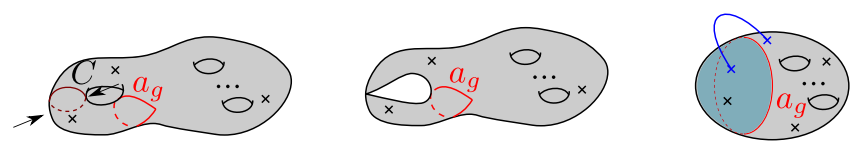

FIG. 5. Integration cycle on the cut surface.

$$
\int_{P}^{z_{i}} \omega_{p_{a}, p_{b}}^{(g-1)}=\left(\int_{z_{0}}^{p_{a}}-\int_{z_{0}}^{p_{b}}\right) \omega_{z_{i}, P}^{(g-1)}
$$

which now has the desired form, if $z_{0}$ is chosen as in (30) and (31). While these terms have been easy to obtain, the ones that descend from degenerating the other terms in the exponent of (19) that contribute to induce a new KobaNielsen factor on the resulting pinched-and-dissected surface are more subtle, and we shall not treat them here but instead focus exclusively on how the propagator $1 / p_{C}^{2}$ is produced.

If now $z_{i}$ is on the other side of the cycle $C$, the path between $P$ and $z_{i}$ is a sum of two segments, as in Fig. 5:

$$
\int_{P}^{z_{i}}=\int_{P}^{z_{b}}+\int_{z_{a}}^{z_{i}}
$$

As seen in the picture, the path can be deformed so as to make apparent that it contains the following integral:

$$
\int_{z_{b}}^{z_{a}} \omega_{p_{a}, p_{b}}^{(g-1)}
$$

Generically, this term is equal to $\tau$, up to subleading corrections of order $O(1)+O(q)$. If we follow the refinement of the plumbing fixture construction developed in [31] called the "funnel formalism," this integral is exactly equal to the lower right entry of the period matrix $\tau$ in Eq. (38); see (3.27) in [31] and [33].

If $I=\left\{i_{1} \ldots i_{k}\right\}$ denotes the set of particles being on the other side of the cycle $C$, from those terms we therefore get a global factor of

$$
4 i \pi \tau \ell_{g} \cdot \sum_{i \in I} k_{i} .
$$

Finally we need to investigate the last category of terms, those of the form $k_{i} \cdot k_{j} \int_{P}^{z_{j}} \omega_{z_{i}, z_{0}}$. But the degeneration is essentially identical to what we did before. When $z_{j}$ is on the same side of the cycle as $P$, nothing happens. If $z_{j}$ is on the other side, we get a factor of $\tau$ for each of these $z_{j}$.

Equivalently, because of Eq. (32) we need the degeneration of the prime form. In [29], the full degeneration of the string world sheet integrals into worldline graphs ("tropical graph") was studied and it was verified that the logarithm of the prime form descends to the worldline propagator of [34]. The latter is given by the sum of the distance in the graph between two points, which essentially parametrizes the degeneration. In field theory, for a graph with an edge of proper time $T$, there always is a modulus of the Riemann surface parametrized by $q=\exp \left(-2 \pi\left(T / \alpha^{\prime}+\theta\right)\right) \rightarrow 0$ for $\theta \in[0 ; 2 \pi[$ such that

$$
\ln (E(x, y))=\ln (q)+\cdots .
$$

If we now look at our case where the surface is degenerated in one cycle, this fact needs to remain true (essentially 
because the limit is continuous and the deformation of this cylinder does not influence the other moduli of the surface to first approximation) and all the propagators $\ln \left(\left|E\left(z_{i}, z_{j}\right)\right|\right)$ that end up splitting apart two punctures on each side of $C$ produce a factor of $\ln (q)$ [35]. If we call $\mathcal{I}$ and $\mathcal{J}$ the (disjoint) sets of punctures on each side of the cut, we get a total factor of

$$
\ln (q) \sum_{i \in \mathcal{I}, j \in \mathcal{J}}\left(k_{i} \cdot k_{j}\right)
$$

To conclude, we can collect all the terms that undergo a degeneration in (19). They conspire to produce a quadratic propagator given by $K^{2}=\left(\ell+\sum k_{i}\right)^{2}$ which appears as follows:

$$
\int_{|q|<\epsilon} \frac{d^{2} q}{|q|^{2}}|q|^{-\alpha^{\prime} \pi K^{2}} \propto \frac{1}{\alpha^{\prime} K^{2}}+O(\epsilon)
$$

where we have used that a $d^{2} \tau \propto d^{2} q /|q|^{2}$ is a modulus of the surface and, hence, is being integrated over in the full string amplitude. It can also be checked that all other dependence on the modulus drops, to subleading order, as far as the exponential is concerned [36].

Using this property in combination with the observation that the cycle running through a node is a topological invariant proves that all the graphs obtained in the tropical or field-theory limit can be given a uniform loop momentum. In the next section we study this labeling and use it in the monodromy relations.

\section{B. Graph labeling in the field-theory limit}

\section{Closed string}

Let us now study the graph labeling induced in the field-theory limit for this closed string picture. The choice of the point where the cycles touch in Fig. 1 defines all the possible degeneration channels and the associated momenta. The graphs are obtained by letting the puncture travel through the whole surface and pinching all possible $a$-type cycles. To identify the momentum flowing through an inner edge, work out which homology cycle being pinched (as in Fig. 8) on the Riemann surface and derive the momentum flowing through it with the rules of Eqs. (34) and (33).

The fact that the punctures move over the whole surface implies in particular that, for a given type of degeneration with prescribed momenta, all the graphs with legs permuted should appear in the integrand.

\section{Open string}

In the open string, the graph labeling that emerges from the integration over the string moduli space is similar to the closed string picture, expect that individual graphs are color ordered. This allows one to select restricted classes of

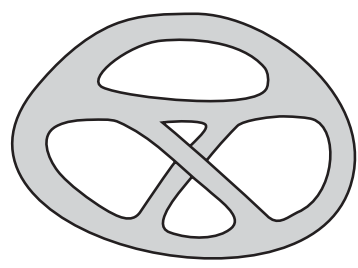

FIG. 6. Non-planar-looking closed string graph.

numerators when studying properties like the monodromy relations.

The open string will be the subject of the next section where we study the monodromy relations at two and three loops. We give there more details and examples on the systematics of the limit and the labeling.

\section{Nonplanar graphs}

There are two types of nonplanar contributions present in the field-theory limit of closed string graphs (in gravity amplitudes): nonplanar vacuum graphs and planar vacuum graphs where external legs are inside. While the latter may seem to cause no troubles concerning the definition of the loop momentum, the former may appear problematic. They are actually not and are neatly generated by the mechanism of the field-theory limit (pinching $a$-cycles); therefore, they also come with a uniquely defined loop momentum. The interested readers can look at the graph in Fig. 6 and convince themselves that the graph suggested by the drawing of this Riemann surface can be obtained from a regular "planar-looking" genus 4 surface by pinching a sum of $a$-cycles with \pm 1 coefficients.

There are nonorientable open-string graphs, and it would be interesting to study the loop momentum of these graphs, too [37].

\section{APPLICATION TO THE MONODROMY RELATIONS IN OPEN-STRING AND GAUGE THEORY AT LOOP LEVEL}

Monodromy relations to all loop orders were derived in [6] in a representation involving loop momentum. Compared to the tree-level case $[9,10]$, the relations do not hold at the level of the amplitude but at the level of the integrand. This stems from the fact that the integrand has both local and global monodromies, and the latter involve phases that depend on the loop momentum. The whole construction is fairly simple and exposed in [6] so it will not be reviewed too deeply here.

The basic idea is to consider a particular open-string loop diagram with particles ordered along the boundaries (inner and outer). Using a representation with loop momentum yields directly an integrand that is holomorphic, as we saw above. Therefore, taking one of these particles along a closed contour inside the surface gives, via the residue theorem, that the sum of all individual portion vanishes exactly at the integrand level. Each portion can be rewritten 

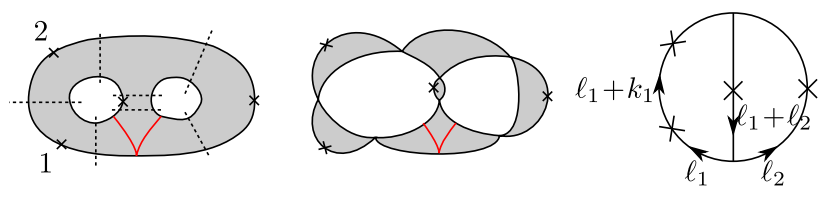

FIG. 7. Two-loop example of field-theory limit for open strings and determination of the loop momentum.

as a properly ordered open-string integrand but at the cost of picking up a phase, that depends on the loop momentum when the particle is on a different boundary than the one we started from. The portions of the contour that run along the $a$-cycles (in red in Fig. 7) cancel after loop-momentum integration (they are related by a simple shift in the loop momentum; see [38] for detailed examples at one loop).

\section{A. Two loops}

To be concrete, we provide an example of the fieldtheory limit of two-loop four-gluon amplitude in type I superstrings.

The orientable topologies of the open-string amplitude (no cross-caps) for $\mathcal{N}=4$ Yang-Mills at two loops are obtained from the celebrated two-loop formulas for closed strings of D'Hoker and Phong [39]. They read

$$
\begin{aligned}
A_{\text {orient }}^{(2)}(1,2,3,4)= & s_{12} s_{23} A^{\text {tree }}(1,2,3,4) \int \frac{\prod_{I \leq J} d \Omega_{I J}}{(\operatorname{det} \operatorname{Im} \Omega)^{5}} \\
& \times \int_{(\partial \Sigma)^{4}} \mathcal{Y}_{S} \exp \left(\sum_{i, j} k_{i} \cdot k_{j} \mathcal{G}\left(z_{i j}\right)\right),
\end{aligned}
$$

up to a global normalization factor, and where $A^{\text {tree }}(1,2$, $3,4)$ is the tree-level four-gluon color-ordered partial amplitude, while the kinematics invariants are defined by $s_{i j}=-\left(k_{i}+k_{j}\right)^{2}$. The integration ordered along the boundary $(\partial \Sigma)^{4} \simeq\left\{\forall i=1 \ldots 4, z_{i} \in \partial \Sigma, z_{1}<z_{2}<z_{3}<z_{4}\right\}$ and $\mathcal{Y}_{S}$ is defined by

$$
\begin{aligned}
3 \mathcal{Y}_{S}= & \left(k_{1}-k_{2}\right) \cdot\left(k_{3}-k_{4}\right) \Delta\left(z_{1}, z_{2}\right) \Delta\left(z_{3}, z_{4}\right)+(13)(24) \\
& +(14)(23),
\end{aligned}
$$

in terms of the differential form bilinears

$$
\Delta(z, w)=\omega_{1}(z) \omega_{2}(w)-\omega_{1}(w) \omega_{2}(z) .
$$

For maximally supersymmetric amplitudes in general at two loops in type I and type II, the field-theory limit procedure was worked out in $[29,40]$ and the numerators are given by the tropical limit of the factor $\mathcal{Y}_{S}$, which equals the kinematic invariant $s_{i j}$ whenever the two legs are on the same $b$-cycle and no subtriangle is present in the graph, which matches the field-theory result of [41] (this means that we just have double-box and nonplanar double-box graphs).
In terms of the string loop integrand $I^{(2)} \simeq I^{(2)}\left(z_{i}, \ell_{i}\right)$ of $A_{\text {orient }}^{(2)}$, the monodromy relations of [6] at two loops read

$$
\begin{aligned}
k_{1} & \cdot k_{2} I^{(2)}(2134)+k_{1} \cdot\left(k_{2}+k_{3}\right) I^{(2)}(2314) \\
& -\ell_{1} \cdot k_{1} I^{(2)}(234|1| \cdot)-\ell_{2} \cdot k_{1} I^{(2)}(234|.| 1) \simeq 0 .
\end{aligned}
$$

The two terms on the rightmost part correspond to nonplanar amplitudes where the particle 1 is integrated along the first and second inner disks of the two-loop open string graph, respectively (from left to right in Fig. 7). The $\simeq$ symbol means "up to terms that vanish after momentum integration." These are generated by integrals along the boundary of the cut surface and correspond to loopmomentum shifts.

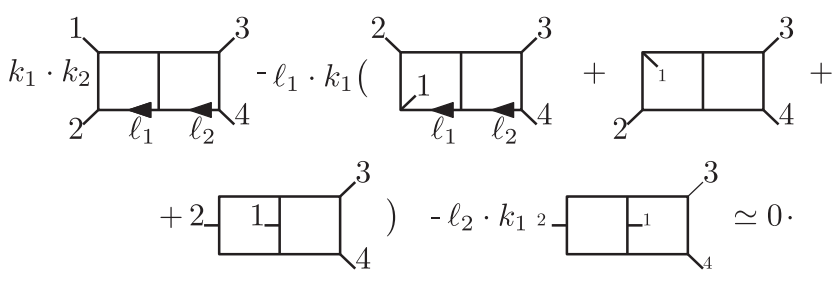

All other diagrams are suppressed by supersymmetry in the field-theory limit because of the properties of the tropical limit of the integrand given by $\mathcal{Y}_{S}$ that we just described. These graphs are scalar graphs, with their denominator and with the same numerator $s_{12}=-\left(k_{1}+k_{2}\right)^{2}$ because $\mathcal{Y}_{s}=s_{34}=s_{12}$ (again see Table 1, p. 41, in Ref. [29]). Therefore, these graphs are just scalar graphs with a constant numerator.

Using the antisymmetry of the three-point vertex $[6,38]$, we can equate the two graphs on the second line up to a sign and reduce the factors in front of the graphs to differences of propagators:

$$
\begin{gathered}
\ell_{1} \cdot k_{1}=\left(\ell_{1}+k_{1}\right)^{2}-\ell_{1}^{2}, \\
\left(\ell_{1}+k_{2}\right) \cdot k_{1}=\left(\ell_{1}+k_{1}+k_{2}\right)^{2}-\left(\ell_{1}+k_{2}\right)^{2}, \\
\left(-\ell_{1}+\ell_{2}\right) \cdot k_{1}=\left(\ell_{1}-\ell_{2}\right)^{2}-\left(\ell_{1}-\ell_{2}-k_{1}\right)^{2} .
\end{gathered}
$$

In this way, six terms are produced which almost cancel pairwise:

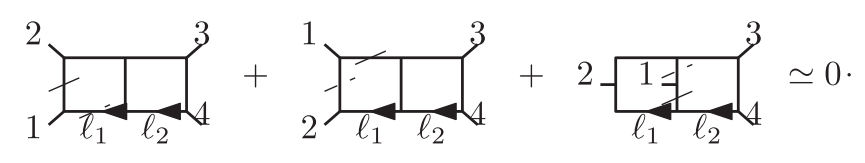

In this equation, the plain (respectively, dashed) lines correspond to a positive (respectively, negative sign). Four terms cancel pairwise, while two, the negative contribution of the first graph and the positive one of the last graph, differ by a shift in the loop momentum as 


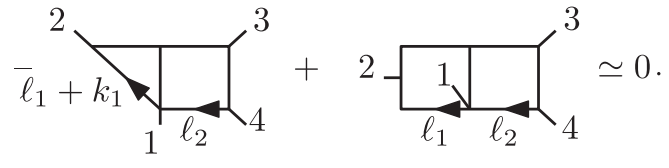

Because the relation is exact at fixed loop momentum, this gives a precise definition the terms in the right-hand side. A more graphical explanation of this phenomenon can be found in [38]. At any rate, after loop-momentum integration, these terms cancel, as they should. Note that for more generic amplitudes, the numerators are not simply constants anymore and the field-theory limit of the terms on the right-hand side could provide interesting physical quantities. These will be studied elsewhere.

This derivation provides a stronger check than the unitarity cut check that was originally performed in [6].

\section{B. A relation at three loops}

What we have seen so far is that the string representation in terms of loop momentum induces a global definition of the loop momentum. Now we will investigate a new phenomenon related to this that arises at three loops: there are two different vacuum topologies of one-particle-irreducible graphs, mercedes and ladder, which share the same loop momentum.

After characterizing this effect, we will work out an example of application of the monodromy relations to support further that their connection to the BCJ colorkinematics duality extends to all loops.

Figure 8 displays two representative graphs that follow from the field-theory limit of the open-string graph on the left-hand side. Both these graphs appear under the same loop-momentum integral in the field-theory limit and provide a natural correspondence between the same loop momentum but in different graphs.

The monodromy relations in string theory at three loops are obtained by following the method exposed in [6]. Circulating leg 1 inside a previously planar graph with ordering $1<2<3<4$ as in Fig. 8 yields a relation, whose field-theory limit is given by

$$
\begin{aligned}
k_{1} & \cdot k_{2} I^{(3)}(2134)+k_{1} \cdot\left(k_{2}+k_{3}\right) I^{(3)}(2314) \\
& -\ell_{1} \cdot k_{1} I^{(3)}(234|1| . \mid .)-\ell_{2} \cdot k_{1} I^{(3)}(234|.| 1 \mid .) \\
& -\ell_{3} \cdot k_{1} I^{(3)}(234|.| . \mid 1) \simeq 0 .
\end{aligned}
$$

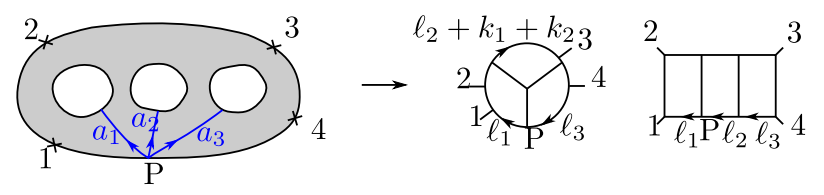

FIG. 8. The two graphs on the right-hand side are generated by the field-theory limit of the open-string graph on the left-hand side. It is an interesting exercise for the reader to work out this example explicitly.
The notations are similar to those of Eq. (54). The terms on the second line correspond to nonplanar amplitudes where particle 1 is integrated along the first, second and third inner disks, respectively, according to the numbering of the $a$ cycles in Fig. 8.

Many graphs arise in this integrand relation [42]. They mix different topologies and orderings. The systematics of the propagator cancellation is similar to what happens at two loops. We illustrate this below for a particular subset of these graphs, which will give stronger evidence that a BCJ representation always satisfies the monodromy relation, up to the loop-momentum shifting terms.

The main point is that this sum of graphs can be reorganized into BCJ triplets. For instance, the following four terms appear in the sum in the left-hand side of (61):

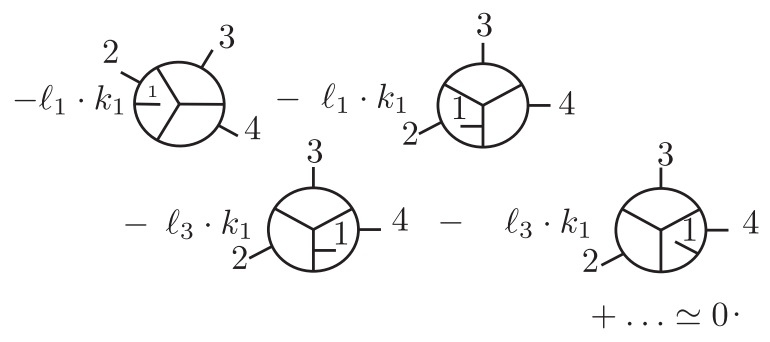

where $+\cdots$ indicate the rest of the terms of the sum. These graphs represent full integrands: numerators over denominator. They are those of any gauge theory we started with in the open string [43].

With the momenta are distributed as in Fig. 8, it is easy to see that the factors in front of the graphs indeed recombine into differences of irreducible propagators which organize themselves as a BCJ identity with shifted momenta of the form
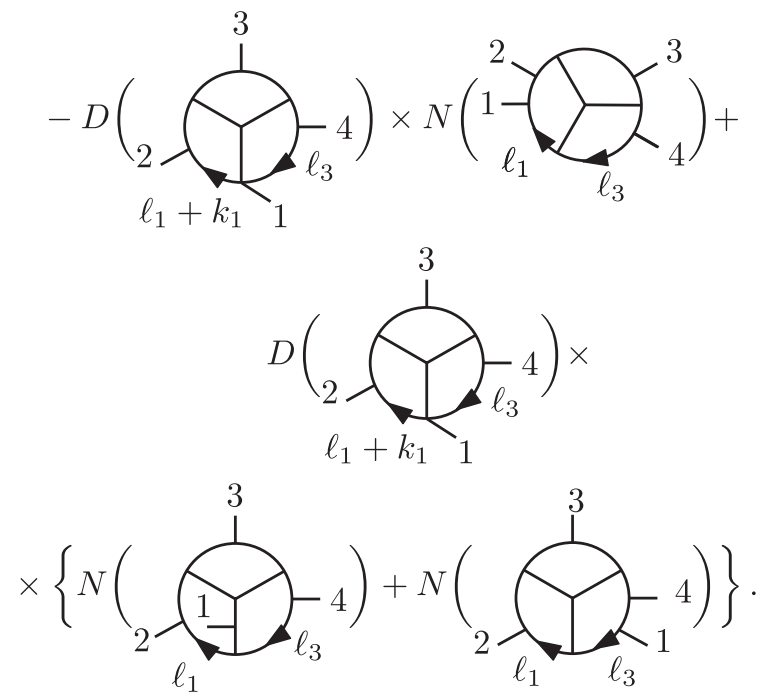

Here, $D(\cdot)$ is the scalar denominator corresponding to the graph (nonobvious loop-momentum locations are depicted, and $\ell_{2}$ is not affected) and $N(\cdot)$ is the numerator of the 
corresponding graph. We have used again the antisymmetry of the three-point vertex

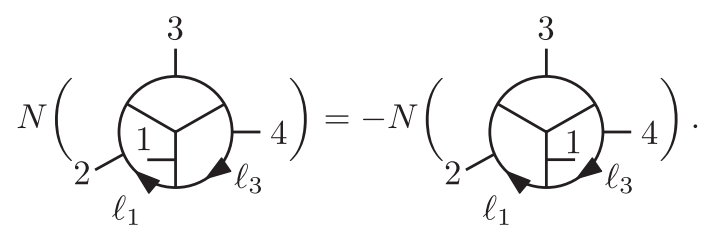

The three terms above therefore combine into a BCJ triplet involving some loop-momentum shifts on top of denominators with one propagator canceled.

We have worked out the specific case that is the most delicate, i.e. the one that involves loop-momentum shifts. The other triplet identities are simpler and therefore it is very reasonable to guess that the property persists for all types of trivalent graphs also including those with internal triangles, bubbles or not-see [38] for examples at one loop.

Note that an identity that would mix up mercedes and ladder topologies requires one to apply the monodromy relations twice or to start from a nonplanar amplitude.

To sum up the relations that stem from the monodromy relations in the field-theory limit, we schematically denote by the letter $J_{G, e}$ the sum of BCJ triplets for the graph $G$ with one inner edge $e$ contracted. We obtain

$$
\sum_{G, e} \frac{J_{G, e}}{D_{G, e}} \simeq 0
$$

In the generalized double-copy construction [44], these $J$ functions generate higher point vertices that need to be canceled by introducing contact terms. The structure of these objects is still quite poorly understood, in particular how to simplify them as much as possible, and it would be interesting to see if the monodromy relations can provide some formal constraints on these objects, maybe in relation to the loop shifting terms of the right-hand side.

\section{DISCUSSION}

\section{A. An integrand in field theory}

In this paper we analyzed some aspects related to the definition of the loop momentum in string and field theory. This formalism was mostly developed to be applied to the monodromy relations, but it would be very interesting to see if the global integrand defined in this way has any nice physical properties.

Furthermore, in standard perturbative field theory there is no particular notion of field-theory integrand except in the case of planar amplitudes: this has led to remarkable constructions such as the all-loop integrand for planar $\mathcal{N}=4$ super-Yang Mills [45] and the amplituhedron [46]. This program was then extended to gravitational theories in [47] and it would be interesting to see if all these constructions are connected to the general considerations presented in this paper.

The ambitwistor string [48], based on the scattering equation formalism [49] also provides loop integrands [26,50-58]. The bottleneck in pushing this formalism to all loops has so far been the understanding of the geometry of the moduli space and the connection to the zero modes (loop momentum) in the path integral. There is no doubt that a better understanding of the loop momentum in string theory should help to fix these issues.

\section{B. Kawai-Lewellen-Tye}

Since they realize splitting of the holomorphic and antiholomorphic degrees of freedom in string integrands, loop-momentum representations should also be linked to the extension of the tree-level Kawai-Lewellen-Tye (KLT) $[59,60]$ formulas to loop level. Recently, Mizera has reformulated in the language of twisted cycles this program $[61,62]$ and a deeper understanding of the loop momentum will be necessary to understand these constructions at loop level where global monodromies arise. Relatedly, it would be very interesting to see if these relations can be extended to amplitude relations. The theory developed in [63] for field-theory integrands, inspired from [61], would seem like a natural starting point to study these questions. Relatedly, "generalized elliptic functions" have been introduced in [64-67] and it would be interesting to see if a proper treatment of the loop momentum can help in characterizing the nature of these objects.

\section{Twisted strings and modular invariance}

Twisted strings [68] are the tensionful versions of ambitwistor strings. To my knowledge, the first time such a construction was mentioned is in the paper [69], and in spirit they were present in $[70,71]$ already. Classically, they are just identical to traditional string theory; but their quantization is modified (different operator ordering), which results in a truncated spectrum. The cleanest way to understand their scattering amplitudes is at tree level so far, via the twisted period relations of [62].

It is conjectured [72] that the loop-level version should also involve only a change in oscillator modes of the string; therefore, all we said here about the loop-momentum zero modes should apply to the twisted string too. However, loop amplitudes have proven difficult to write so far, and a very good hope to guess them would be to generalize the twisted period relations to loop level. This ties in with the previous paragraph on KLT.

One could even think of using these twisted string loop amplitudes to then take the ambitwistor string limit (tensionless limit of the twisted string; see [72-76]). But one may doubt that this could produce a sensible answer, mostly because the loop momentum breaks modular invariance [4] and the saddle point equations of the tensionless 
limit [77] seem to induce a maximum value for the loop momentum, while all values should be allowed and integrated on to give back the original integral without loop momentum. This problem will be studied elsewhere.

\section{ACKNOWLEDGMENTS}

I thank David Andriot, Enrico Hermann, Alexander Ochirov, Julio Parra-Martinez, Boris Pioline, Amit Sever and Sasha Zhiboedov for useful discussions and comments. This research project has been supported by a Marie Skłodowska-Curie Individual Fellowship of the European Commissions Horizon 2020 Program under Contract No. 749793 NewLoops.

Note added.-Since this paper has appeared, another check on the prescription was provided in [78], in the nonplanar sector, where the phases of the subleading order relations found in [6] were corrected to match those of [7].

\section{APPENDIX: DEFINITIONS}

Here are the conventions that are used in this paper (we follow mostly Kiritsis [79]):

$$
\begin{gathered}
z=\sigma^{1}+i \sigma^{2}, \quad \bar{z}=\sigma^{1}-i \sigma^{2}, \\
\frac{\partial}{\partial z}=\frac{1}{2}\left(\partial_{1}-i \partial_{2}\right), \quad \frac{\partial}{\partial \bar{z}}=\frac{1}{2}\left(\partial_{1}+i \partial_{2}\right) .
\end{gathered}
$$

The metric reads $d^{2} s=2 g_{z \bar{z}} d z d \bar{z}$; therefore, $\sqrt{g}=g_{z \bar{z}}$ such that the volume measure is given by $\int \sqrt{g_{a b}} d^{2} \sigma=$ $\int \sqrt{g} d^{2} z$, which yields

$$
2 d \sigma_{1} d \sigma_{2}=d^{2} z=i d z \wedge d \bar{z} .
$$

For the diagonal metric $g_{a b}=\operatorname{diag}(1,1)$ we have $g_{z \bar{z}}=1 / 2$, which implies that $\delta^{2}(z, \bar{z})=\frac{1}{2 \sqrt{g}} \delta(x) \delta(y)=\delta(x) \delta(y)$, as this yields

$$
\int \sqrt{g} d^{2} z \delta^{2}(z, \bar{z})=1
$$

We also have

$$
\partial_{\bar{z}} \frac{1}{z}=\partial_{z} \frac{1}{\bar{z}}=2 \pi \delta^{2}(z, \bar{z})
$$

We will use the language of differential forms (all conventions are spelled out in the Appendix), upon which, essentially,

$d^{2} z=i d z \wedge d \bar{z}, \quad \partial=\partial_{z} d z, \quad \bar{\partial}=\partial_{\bar{z}} d \bar{z}, \quad d=\partial+\bar{\partial}$,

where $d$ is the standard differential operator, $d^{2}=0$. The $i$ normalization factor will be important soon. The Stokes theorem states that, for $\omega$ a $k$-form and $D$ a $(k+1)$-chain, we have

$$
\int_{\partial D} \omega=\int_{D} d \omega
$$

where $\partial D$ is the boundary of $D$.

The prime form is a $(-1 / 2,0) \otimes(-1 / 2,0)$ biholomorphic form defined on the universal covering of the surface by

$$
E(x, y)=\frac{\theta[\nu]\left(\int_{x}^{y}\left(\omega_{1}, \ldots, \omega_{g}\right) \mid \Omega\right)}{h_{\nu}(x) h_{\nu}(y)} \in \mathbb{C},
$$

where $h_{\nu}(x)^{2}=\sum_{I} \omega_{I} \partial_{I} \theta[\nu](0 \mid \Omega)$ are half-differentials (section of the square root of the canonical bundle). It is independent of the spin structure chosen to define it.

The Riemann theta functions are defined by

$$
\theta[\nu](\zeta \mid \Omega)=\sum_{n \in \mathbb{Z}^{g}} e^{i \pi(n+\beta) \cdot \Omega(n+\beta)} e^{2 i \pi(n+\beta) \cdot(\zeta+\alpha)},
$$

where $\left[\begin{array}{l}\beta \\ \alpha\end{array}\right]=\nu \in\left(\mathbb{Z} / \mathbb{Z}_{2}\right)^{2 g}$ is a theta characteristic. They have monodromies that can be found in standard textbooks, which lead to the following monodromies for the prime form [4]:

$$
E(x, y) \rightarrow \exp \left(-\Omega_{J J} / 2-\int_{x}^{y} \omega_{J}\right) E(x, y)
$$

and trivial signs along $a$-cycles.
[1] J. A. Shapiro, Loop graph in the dual tube model, Phys. Rev. D 5, 1945 (1972).

[2] E. P. Verlinde and H. L. Verlinde, Chiral bosonization, determinants and the string partition function, Nucl. Phys. B288, 357 (1987).
[3] E. P. Verlinde and H. L. Verlinde, Multiloop calculations in vovariant superstring theory, Phys. Lett. B 192, 95 (1987).

[4] E. D'Hoker and D.H. Phong, The geometry of string perturbation theory, Rev. Mod. Phys. 60, 917 (1988). 
[5] E. D'Hoker and D. H. Phong, Conformal scalar fields and chiral splitting on superriemann surfaces, Commun. Math. Phys. 125, 469 (1989).

[6] P. Tourkine and P. Vanhove, Higher-Loop Amplitude Monodromy Relations in String and Gauge Theory, Phys. Rev. Lett. 117, 211601 (2016).

[7] S. Hohenegger and S. Stieberger, Monodromy relations in higher-loop string amplitudes, Nucl. Phys. B925, 63 (2017).

[8] E. Plahte, Symmetry properties of dual tree-graph $N$-point amplitudes, Nuovo Cimento A 66, 713 (1970).

[9] N. E. J. Bjerrum-Bohr, P. H. Damgaard, and P. Vanhove, Minimal Basis for Gauge Theory Amplitudes, Phys. Rev. Lett. 103, 161602 (2009).

[10] S. Stieberger, Open \& closed vs. pure open string disk amplitudes, arXiv:0907.2211.

[11] Z. Bern, J. J. M. Carrasco, and H. Johansson, New relations for gauge-theory amplitudes, Phys. Rev. D 78, 085011 (2008).

[12] Z. Bern, J. J. M. Carrasco, and H. Johansson, Perturbative Quantum Gravity as a Double Copy of Gauge Theory, Phys. Rev. Lett. 105, 061602 (2010).

[13] Z. Bern, J. J. Carrasco, W.-M. Chen, A. Edison, H. Johansson, J. Parra-Martinez, R. Roiban, and M. Zeng, Ultraviolet properties of $\mathcal{N}=8$ supergravity at five loops, Phys. Rev. D 98, 086021 (2018).

[14] Z. Bern, J. J. Carrasco, M. Chiodaroli, H. Johansson, and R. Roiban, The duality between color and kinematics and its applications, arXiv:1909.01358.

[15] B. Feng, Y. Jia, and R. Huang, Relations of loop partial amplitudes in gauge theory by unitarity cut method, Nucl. Phys. B854, 243 (2012).

[16] B. Feng, R. Huang, and Y. Jia, Gauge amplitude identities by on-shell recursion relation in S-matrix program, Phys. Lett. B 695, 350 (2011).

[17] R. H. Boels and R. S. Isermann, New relations for scattering amplitudes in Yang-Mills theory at loop level, Phys. Rev. D 85, 021701 (2012).

[18] R. H. Boels and R. S. Isermann, Yang-Mills amplitude relations at loop level from non-Adjacent BCFW shifts, J. High Energy Phys. 03 (2012) 051.

[19] Y.-J. Du and H. Luo, On general BCJ relation at one-loop level in Yang-Mills theory, J. High Energy Phys. 01 (2013) 129.

[20] M. Chiodaroli, M. Gunaydin, H. Johansson, and R. Roiban, Explicit formulae for Yang-Mills-Einstein amplitudes from the double copy, J. High Energy Phys. 07 (2017) 002.

[21] M. B. Green, J. H. Schwarz, and E. Witten, Superstring Theory, Cambridge Monographs on Mathematical Physics Vol. 2 (Cambridge University Press, Cambridge, England, 2012).

[22] Another intuitive picture is that one cannot put a single electric charge at rest on a compact Riemann surface; a second one needs to be added to cancel the charge or a background charge should be included. This background charge breaks the holomorphy of the Green's function.

[23] D. P. Skliros, E. J. Copeland, and P. M. Saffin, Highly excited strings I: Generating function, Nucl. Phys. B916, 143 (2017).

[24] Typical vertex operators for string states would also have a polynomial dependence on $\partial_{z} X$, ghost fields, and other matter fields, in generic string models.
[25] For a standard reference, see [80].

[26] E. Casali and P. Tourkine, Infrared behaviour of the oneloop scattering equations and supergravity integrands, J. High Energy Phys. 04 (2015) 013.

[27] M. Bianchi and A. Sagnotti, Open strings and the relative modular group, Phys. Lett. B 231, 389 (1989).

[28] J. Scherk, Zero-slope limit of the dual resonance model, Nucl. Phys. B31, 222 (1971).

[29] P. Tourkine, Tropical amplitudes, Ann. Henri Poincare 18, 2199 (2017).

[30] J. D. Fay, Theta Functions on Riemann Surfaces (Springer, New York, 1973), Vol. 352.

[31] E. D'Hoker, M. B. Green, and B. Pioline, Higher genus modular graph functions, string invariants, and their exact asymptotics, Commun. Math. Phys. 366, 927 (2019).

[32] See e.g. [[80] III.7]—our $\omega_{P Q}$ forms are denoted $\tau_{P Q}$ there.

[33] The extra bits of the contour add up to create what becomes the Koba-Nielsen factor on the cut surface, which are not in the scope of this paper as we said above.

[34] P. Dai and W. Siegel, Worldline Green functions for arbitrary Feynman diagrams, Nucl. Phys. B770, 107 (2007).

[35] It would be interesting to use the funnel formalism developed in [31] to prove this fact directly.

[36] Other terms may appear in front of the plane waves, when scattering gravitons, for instance, but their presence only affects the numerators of the field theory graphs, not the propagators.

[37] For a study of the monodromy relation for nonorientable at one loop, see [7].

[38] A. Ochirov, P. Tourkine, and P. Vanhove, One-loop monodromy relations on single cuts, J. High Energy Phys. 10 (2017) 105.

[39] E. D'Hoker and D. H. Phong, Lectures on two loop superstrings, Conf. Proc. C0208124, 85 (2002), https://arxiv.org/ abs/hep-th/0211111.

[40] M. B. Green, J. G. Russo, and P. Vanhove, Modular properties of two-loop maximal supergravity and connections with string theory, J. High Energy Phys. 07 (2008) 126.

[41] Z. Bern, J. S. Rozowsky, and B. Yan, Two loop four gluon amplitudes in $\mathcal{N}=4$ Superyang-Mills, Phys. Lett. B 401, 273 (1997).

[42] Counting by hand graphs with no triangles (having in mind $N=4$ super-Yang-Mills) give an $\leq O(150)$ graphs.

[43] One strength of the monodromy relations is that they are universal.

[44] Z. Bern, J. J. M. Carrasco, W.-M. Chen, H. Johansson, R. Roiban, and M. Zeng, Five-loop four-point integrand of $N=8$ supergravity as a generalized double copy, Phys. Rev. D 96, 126012 (2017).

[45] N. Arkani-Hamed, J. L. Bourjaily, F. Cachazo, S. CaronHuot, and J. Trnka, The all-loop integrand for scattering amplitudes in planar $\mathcal{N}=4$ sym, J. High Energy Phys. 01 (2011) 041.

[46] N. Arkani-Hamed and J. Trnka, The amplituhedron, J. High Energy Phys. 10 (2014) 030.

[47] E. Herrmann and J. Trnka, Gravity on-shell diagrams, J. High Energy Phys. 11 (2016) 136.

[48] L. Mason and D. Skinner, Ambitwistor strings and the scattering equations, J. High Energy Phys. 07 (2014) 048. 
[49] F. Cachazo, S. He, and E. Y. Yuan, Scattering of massless particles: Scalars, gluons and gravitons, J. High Energy Phys. 07 (2014) 033.

[50] T. Adamo, E. Casali, and D. Skinner, Ambitwistor strings and the scattering equations at one loop, J. High Energy Phys. 04 (2014) 104.

[51] K. Ohmori, Worldsheet geometries of Ambitwistor string, J. High Energy Phys. 06 (2015) 075.

[52] Y. Geyer, L. Mason, R. Monteiro, and P. Tourkine, Loop Integrands for Scattering Amplitudes from the Riemann Sphere, Phys. Rev. Lett. 115, 121603 (2015).

[53] Y. Geyer, L. Mason, R. Monteiro, and P. Tourkine, One-loop amplitudes on the Riemann sphere, J. High Energy Phys. 03 (2016) 114.

[54] F. Cachazo, S. He, and E. Y. Yuan, One-loop corrections from higher dimensional tree amplitudes, J. High Energy Phys. 08 (2016) 008.

[55] S. He and E. Y. Yuan, One-loop scattering equations and amplitudes from forward limit, Phys. Rev. D 92, 105004 (2015).

[56] S. He, R. Monteiro, and O. Schlotterer, String-inspired BCJ numerators for one-loop MHV amplitudes, J. High Energy Phys. 01 (2016) 171.

[57] Y. Geyer, L. Mason, R. Monteiro, and P. Tourkine, Twoloop scattering amplitudes from the Riemann sphere, Phys. Rev. D 94, 125029 (2016).

[58] Y. Geyer and R. Monteiro, Two-loop scattering amplitudes from ambitwistor strings: From genus two to the Nodal Riemann sphere, J. High Energy Phys. 11 (2018) 008.

[59] H. Kawai, D. C. Lewellen, and S. H. H. Tye, A relation between tree amplitudes of closed and open strings, Nucl. Phys. B269, 1 (1986).

[60] N. E. J. Bjerrum-Bohr, P. H. Damgaard, T. Sondergaard, and P. Vanhove, The momentum kernel of gauge and gravity theories, J. High Energy Phys. 01 (2011) 001.

[61] S. Mizera, Combinatorics and topology of Kawai-LewellenTye relations, J. High Energy Phys. 08 (2017) 097.

[62] S. Mizera, Scattering Amplitudes from Intersection Theory, Phys. Rev. Lett. 120, 141602 (2018).

[63] P. Mastrolia and S. Mizera, Feynman integrals and intersection theory, J. High Energy Phys. 02 (2019) 139.

[64] C. R. Mafra and O. Schlotterer, Double-Copy Structure of One-Loop Open-String Amplitudes, Phys. Rev. Lett. 121, 011601 (2018).
[65] C. R. Mafra and O. Schlotterer, Towards the N-point oneloop superstring amplitude I: Pure spinors and superfield kinematics, J. High Energy Phys. 08 (2019) 090.

[66] C. R. Mafra and O. Schlotterer, Towards the N-point one-loop superstring amplitude III: One-loop correlators and their double-copy structure, J. High Energy Phys. 08 (2019) 092.

[67] C. R. Mafra and O. Schlotterer, Towards the N-point one-loop superstring amplitude II: Worldsheet functions and their duality to kinematics, J. High Energy Phys. 08 (2019) 091.

[68] Also called "left-handed" [72] or "chiral strings" [73,74]. The "twisted string" terminology was used in [81] because toroidal compactifications allows what are chiral strings in flat space to acquire nontrivial excitations; hence, they are not really chiral.

[69] S. Hwang, R. Marnelius, and P. Saltsidis, A general BRST approach to string theories with Zeta function regularizations, J. Math. Phys. (N.Y.) 40, 4639 (1999).

[70] J. Gamboa, C. Ramirez, and M. Ruiz-Altaba, Null spinning strings, Nucl. Phys. B338, 143 (1990).

[71] J. Gamboa, C. Ramirez, and M. Ruiz-Altaba, Quantum null (super)strings, Phys. Lett. B 225, 335 (1989).

[72] W. Siegel, Amplitudes for left-handed strings, arXiv:1512 .02569

[73] M. M. Leite and W. Siegel, Chiral closed strings: Four massless states scattering amplitude, J. High Energy Phys. 01 (2017) 057.

[74] Y.-t. Huang, W. Siegel, and E. Y. Yuan, Factorization of chiral string amplitudes, J. High Energy Phys. 09 (2016) 101.

[75] E. Casali, Y. Herfray, and P. Tourkine, The complex null string, Galilean conformal algebra and scattering equations, J. High Energy Phys. 10 (2017) 164.

[76] E. Casali and P. Tourkine, On the null origin of the Ambitwistor string, J. High Energy Phys. 11 (2016) 036.

[77] D. J. Gross and P. F. Mende, String theory beyond the Planck scale, Nucl. Phys. B303, 407 (1988).

[78] E. Casali, S. Mizera, and P. Tourkine, Monodromy relations from twisted homology, J. High Energy Phys. 12 (2019) 087.

[79] E. Kiritsis, String Theory in a Nutshell (Princeton University Press, Princeton, 2007).

[80] H. M. Farkas and I. Kra, Riemann Surfaces (SpringerVerlag, Berlin, 1980), Vol. 71.

[81] E. Casali and P. Tourkine, Winding modes of tensionful ambitwistor strings, arXiv:1710.0124. 